\title{
Sarcopenic obesity and its association with dietary intake among Brazilian adults clinically screened to participate in a lifestyle changing program
}

\author{
K. C. P. McLellan ${ }^{1}$, E. P. de Oliveira ${ }^{1}$, J. E. Corrente ${ }^{2}$, R. C. Burini ${ }^{1}$ \\ ${ }^{1}$ Department of Public Health and ${ }^{2}$ Biosciences, Medical School, Sao Paulo State University - UNESP, Brazil
}

\begin{abstract}
Sarcopenia has multiple contributing factors and among them inadequate diet, sedentary lifestyle, and chronic diseases ${ }^{(1)}$. Based on increased awareness of sarcopenia it is important to identify dietary strategies, lifestyle changes and treatments that can prevent or delay the onset of sarcopenia ${ }^{(2)}$. The aim of the present study was identify the association of sarcopenic obesity with dietary intake among Brazilian adults.

A cross-sectional study was conducted with 580 adults ( $80.3 \%$ women and mean age of $53.5 \pm 10.55$ years) clinically screened to participate in a lifestyle changing program. Anthropometric profile, biochemical evaluation and dietary intake were assessed for all participants. Fasting blood sample was used for biochemical analysis. Participants were classified as sarcopenic obese based on a higher quartile of waist circumference and lower quartile of muscle mass index (by BIA). Metabolic Syndrome (MS) was diagnosed using NCEP ATPIII (2001) criteria. Logistic regression (Odds ratio) was performed in order to observe the association of waist circumference and muscle mass index with dietary intake and metabolic syndrome. The prevalence of sarcopenic obesity was $1.5 \%$ among the studied population. An adequate intake of legumes ( $\mathrm{OR}=0.47 ; 0.24-0.93)$, and $\mathrm{HEI}(\mathrm{OR}=0.308 ; 0.09-0.98)$ were associated with a protection for higher waist circumference measurement while inadequate intake of total fat $(\mathrm{OR}=2.31 ; 1.18-4.51)$, and fiber $(\mathrm{OR}=1.97 ; 1.05-3.86)$ were associated with a risk factor for higher waist circumference. Muscle mass index was associated with adequate intake of fruit $(\mathrm{OR}=0.46 ; 0.25-0.85)$; meat $(\mathrm{OR}=0.45 ; 0.20-0.99)$, dietary fiber $(\mathrm{OR}=0.48 ; 0.27-0.83)$, and $\mathrm{HEI}(\mathrm{OR}=0.38 ; 0.16-0.94)$. In conclusion, an adequate intake of legumes, fruit, dietary fiber, meat and HEI might be beneficial to prevent or delay the onset sarcopenic obesity in this population.
\end{abstract}

This study was supported by CNPq, Brazil.

1. Cruz-Jentoft AJ et al. (2010) Age and Ageing 39, 412-423.

2. Waters DL et al. (2011) Clin Geriatr Med 27, 401-421. 